\title{
Improved near-term Earth rotation predictions using atmospheric angular momentum analysis and forecasts
}

\author{
Thomas J. Johnson ${ }^{\mathrm{a}, *}$, Brian J. Luzum ${ }^{\mathrm{b}}$, Jim R. Ray ${ }^{\mathrm{c}}$ \\ ${ }^{a}$ Earth Orientation Department, U.S. Naval Observatory, Washington, DC, USA \\ ${ }^{\mathrm{b}}$ Civil and Coastal Engineering, University of Florida, Gainesville, FL, USA \\ ${ }^{c}$ NGS, National Oceanic and Atmospheric Administration, Silver Springs, MD, USA
}

Received 23 July 2004; received in revised form 22 October 2004; accepted 25 October 2004

\begin{abstract}
Predicted values of atmospheric angular momentum (AAM) from the U.S. National Centers for Environmental Prediction (NCEP) were introduced into the International Earth Rotation and Reference Systems Service (IERS) Bulletin A (rapid service/prediction of Earth orientation) combination during 2000 in an effort to improve the nearterm universal time-coordinated universal time (UT1-UTC) predictions. In this approach, only the atmospheric contributions to the excitation of UT1 variations were included and contributions from other sources, such as the oceans, were neglected. However, we observed that using this approach the AAM inputs sometimes degraded the UT1 predictions as much as they helped and suspended their use in early 2001 for re-evaluation. This re-evaluation resulted in a greater appreciation of the systematic differences between the AAM and UT1R series as well as suggests that, in the actual Earth system, the oceans balance some of the atmospheric variability on specific time scales between 2 and 15 days. Since near-real time oceanic angular momentum information is currently not available, an improved approach for assimilating AAM data products was developed. These improvements include the use of both pressure and wind AAM terms, use of the previously unavailable AAM analysis data at the 24-h epoch for the latest AAM analysis file, smoothing of the AAM-based Length of day (LOD) to reduce sub-diurnal variability before integrating to UT1R, and removal of a best-fit sinusoid from the AAM UT1R time series. The new procedure reduces the effects of systematic trends (both periodic and linear) that do not appear to be present in actual UT1R variability. Retrospective studies indicate that including this new AAM-derived series may reduce UT1R prediction errors by $\sim 41 \%$ at 5 days into the future. Consequently, AAM-derived estimates of UT1R were restored to IERS Bulletin A starting in the 7 August 2001 issue. Comparisons made after the addition of UTAAM into the Bulletin
\end{abstract}

\footnotetext{
* Corresponding author. Tel.: +1 202762 1518; fax: +1 2027621563 .

E-mail address: johnson.thomas@usno.navy.mil (T.J. Johnson).
} 
A UT1-UTC combination solution indicate a better than 50\% reduction in prediction errors at 5 and 10 days into the future and an $\sim 1 \mathrm{~ms}$ improvement at 30 days into the future.

Published by Elsevier Ltd.

Keywords: Earth rotation; Atmosphere; Angular momentum; Length of day; Earth orientation prediction

\section{Background}

The Earth's orientation is described using five parameters. The longitude and obliquity of the celestial ephemeris pole define the orientation of the Celestial Ephemeris Pole (CEP) with respect to the celestial reference frame. The International Earth Rotation and Reference Systems Service (IERS) makes available the models describing these angles. These models have some deficiencies and therefore they require constant monitoring with the differences between the models and observations reported as "celestial pole offsets." The orientation of the CEP with respect to the terrestrial reference frame is described by the polar motion (PM) parameters $x$ and $y$, while the measure of the angle about the rotation axis through which the solid Earth has rotated is defined as universal time (UT1). UT1R is obtained by correcting the UT1 for the well-known tidal effects (Yoder et al., 1981; McCarthy, 1996).

Out of all five of these Earth's orientation parameters (EOP), UT1 is the most difficult to determine in a real-time estimation process. This is due to the large rapid and seemingly unpredictable day-to-day variations in UT1. UT1 is typically expressed with respect to a reference time defined by atomic clocks, such as coordinated universal time (UTC). UTC differs from the International Atomic Time (TAI) by an integral number of seconds that are introduced to maintain UTC within \pm 0.90 s of UT1.

In the absence of observational data, our knowledge of UT1 degrades rapidly due to stochastic excitation by geophysical forcing. The primary source of UT1 excitation, for periods less than a year, is the exchange of angular momentum between the atmosphere and the Earth's crust. Morabito et al. (1988) analyzed the power-law behavior of geodetic observations and inferred a growth of UT1R error as an integrated random walk process.

In addition, to these five parameters, Length of day (LOD) variations are useful in the study of Earth rotation. LOD variations represent changes in the Earth's rotation rate. LOD is related to the first derivative of UT1-TS with respect to time where TS is a uniform reference time standard. TAI and UTC are examples of uniform time standards. See Table 1 for a complete listing of acronyms used.

More recent studies (Bell et al., 1991; Freedman et al., 1994) have examined the usefulness of atmospheric model estimates of atmospheric angular momentum (AAM) to improve near-term UT1-UTC predictions. Freedman et al. (1994) used a Kalman filter technique to show that the use of AAM wind terms from the U.S. National Meteorological Center's analysis and forecast models improved their nearterm UT1 predictions. The RMS of the UT1 prediction errors for their rapid turnaround product at 5 days into the future could be reduced to $\sim 890 \mu \mathrm{s}$ with the introduction of AAM wind terms. This was a $20 \%$ improvement compared with no AAM data products. However, the study was limited by the accuracy of the data sets available and long data latency, limitations which have greatly improved for the current IERS Bulletin A. The years since the study by Freedman et al. (1994) have seen improvements in the accuracies of very long baseline interferometry UT1 measurements as well as $\sim 50 \%$ reduction in the time between making the observation and the availability of results $(\sim 3$ days). Furthermore, today's Bulletin 
Table 1

Definitions of acronyms used in text

\begin{tabular}{|c|c|}
\hline Acronym & Definition \\
\hline AAM & Atmospheric angular momentum \\
\hline ARIMA & Auto-regressive integrated moving average \\
\hline C04 UT1R & UT1R from the IERS C04 EOP series \\
\hline CEP & Celestial ephemeris pole \\
\hline ECCO & Estimating the circulation and climate of the ocean model \\
\hline EOP & Earth orientation parameters \\
\hline GPS & Global positioning system \\
\hline IB & Inverted barometer \\
\hline IERS & International Earth rotation and reference systems service \\
\hline LOD & Length of day \\
\hline LODR & LOD corrected for tides \\
\hline MRF/AVN & Medium-range forecast/aviation global analysis/forecast system \\
\hline NCEP & National centers for environmental prediction \\
\hline OAM & Oceanic angular momentum \\
\hline PM & Polar motion \\
\hline POCM4B & Parallel ocean climate model run 4B \\
\hline RMS & Root-mean square \\
\hline TAI & International atomic time \\
\hline TS & Uniform reference time standard \\
\hline USNO & United States naval observatory \\
\hline UT1 & Earth's axial rotation angle \\
\hline UT1-UTC & Variations in UT1 with respect to the time standard UTC \\
\hline UT1R & UT1 corrected for tides \\
\hline UT1R-UTC & Variations in UT1R with respect to the time standard UTC \\
\hline UTAAM & UT1-like observation determined using AAM \\
\hline UTC & Coordinated universal time \\
\hline UTGPS & UT1-like observation determined using GPS \\
\hline
\end{tabular}

uses a daily Global Positioning System (GPS) UT1-like observation (UTGPS) that is available with $\sim 1$ day delay (Kammeyer, 2000; Luzum et al., 2001).

In previous, unpublished studies at the U.S. Naval Observatory (USNO), AAM forecast information (no analysis fields) was introduced into an auto-regressive integrated moving average (ARIMA) prediction process. No significant improvement in the UT1 predictions was found. Typically, the USNO prediction errors are $\sim 750 \mu \mathrm{s}$ at 5 days into the future when UTGPS is available and the addition of AAM forecast data did not reduce these errors. This discrepancy with the findings of Freedman et al. (1994) could be due to differences in the ARIMA methodology compared to the Kalman filtering approach and/or to the higher quality, lower latency geodetic data available during the USNO study, especially after introducing daily UTGPS.

Continuing advances in modern global atmospheric models precipitated a re-examination of the utility of AAM to improve near-term UT1-UTC predictions. Our initial 3-month study examined the effects of introducing a 10-day AAM time series created by the combination of the five most recent AAM analysis files with a five-day forecast of AAM. This combination process simply removed the bias between the analysis and the forecast data sets. This study also examined the performance of the AAM values computed with and without the application of the Inverted Barometer (IB) correction. The 10-day window 
was chosen because the ARIMA process has better performance when it has AAM information for a period symmetric with respect to the solution epoch. This study indicated that using the IB corrected AAM in the IERS Bulletin A combination (e.g., IERS, 1998) would improve our UT1 predictions. However, in late 2000, we observed that the AAM-based values sometimes degraded the UT1 predictions as much as they helped. We suspended the use of AAM data at the beginning of 2001 to re-evaluate our entire AAM process.

For this re-evaluation, we tested the performance of the AAM-derived UT1R-like observation (called UTAAM) for a 6-month period in 2001. We investigated the AAM variability, the method used for combining the AAM analysis and forecast data into a homogenous time series, the processing of the AAM to derive UTAAM, and the cause of this degradation in UT1 prediction. The results of this reevaluation are presented together with the new UTAAM processing method incorporated into Bulletin A and preliminary estimates of the new Bulletin accuracies for UT1-UTC.

\section{UT1-UTC predictions}

To predict UT1-UTC, USNO uses a simple ARIMA model. This model was chosen after a number of empirical studies (McCarthy and Luzum, 1991). This model is based upon the assumption that once the known signals are accounted for the resulting time series represents a nonstationery stochastic process. In practice, the prediction of UT1-UTC requires the removal of seasonal effects, solid Earth zonal tides, the offset between UTC and TAI, and discontinuities such as leaps seconds, from the UT1-UTC time series. The prediction of UT1-UTC $n$ days into the future is computed using

$$
(\mathrm{UT} 1 \mathrm{R}-\mathrm{TAI})_{n}=2(\mathrm{UT} 1 \mathrm{R}-\mathrm{TAI})_{0}-(\mathrm{UT} 1 \mathrm{R}-\mathrm{TAI})_{-n}
$$

where $n$ indicates the number of days into the future, $-n$ indicates the number of days in the past, and 0 indicates the epoch of the solution. Prior to being used in this model, the UT1R-TAI time series is smoothed using a Gaussian filter with a variable weight. Empirical testing showed that the long-term predictions would benefit from heavy smoothing of the past observations while short-term predictions would not (McCarthy and Luzum, 1991; Luzum et al., 2001). Therefore, a Gaussian filter with a smoothing constant that is a function of the number of days into the future

$$
\left\langle f\left(t_{0}\right)\right\rangle=\sum_{i} \frac{w\left(t_{i}-t_{0}\right) f\left(t_{i}-t_{0}\right)}{w\left(t_{i}-t_{0}\right)}
$$

where $w\left(t_{i}-t_{0}\right)=\exp \left(t_{i}-t_{0}\right) /(n / 2.35)^{2}$ was chosen. This smoothing reduces the effects of small fluctuations and small errors. Therefore, the long-term predictions are improved since they are responding more to general trends in the data.

\section{Data products}

Several IERS Bulletin A UT1-UTC data products were used in this study. The daily IERS Bulletin A geodetic-only UT1R solution was used for the AAM processing (see next section), and the geodeticonly UT1R solution and predictions were used to compare with the AAM performance. These series 
are denoted "Daily UT1R." For the Earth's actual variations in UT1, we used retrospectively a similar Bulletin A geodetic-only UT1R solution produced more than 2 weeks after the 6-months study period. This avoids the small variations in UT1-UTC around the epoch of the solution due to assimilation of late arrival data. This time series is denoted "Final UT1R."

NCEP uses their Operational Medium-Range Forecast/Aviation Global Analysis/Forecast System (MRF/AVN) to produce two types of AAM data files. The system generates a daily analysis data set that represents the assimilation of globally distributed atmospheric observations as well as a daily 5-day forecast file containing predictions of atmospheric conditions. It should be noted that during this study the MRF/AVN system was upgraded (see Moorthi et al., in press for more details) and these changes were observable in the AAM data products, as discussed in Section 4. Since January 2001, the AAM analysis files contain data at $0,6,12,18$, and $24 \mathrm{~h}$ epochs for the previous day. This is an improvement over earlier analysis products that did not contain information at the 24-h epoch, leaving a data gap between the analysis and forecast series. The 5-day forecast files contain a prediction of AAM every $12 \mathrm{~h}$ starting at $12 \mathrm{~h}$ and ending at $120 \mathrm{~h}$. These datasets are available through the IERS Global Geophysical Fluids Center's Special Bureau of the Atmosphere website <http://www.aer.com/sba.html>.

\section{AAM reanalysis and new processing}

Examination of the UTAAM variability showed systematic trends (both periodic and linear) that do not appear to be present in actual UT1 variability. We noted broadband quasi-periodic effects near 10 days as well as increases in the prediction errors that appeared to be related to the day of the week. Fig. 1b shows the quasi-periodic variations in the differences between the UTAAM and geodetic-only Final UT1R, after the removal of daily linear trends. The quasi-periodic variability, not present in the actual UT1, appeared to degrade the AAM performance for UT1 prediction. Therefore, it was decided that any new technique that would allow UTAAM to be useful in the determination and prediction of UT1 would have to reduce or remove these systematic effects.

After observing the residual variability, the length of the UTAAM time series used in the IERS Bulletin A combination was increased to 15 days (10 days of the most recent analysis files and a 5-day forecast file) to allow for the estimation and removal of quasi-periodic variability. To create this time series, 11 of the most recent analysis files were used together with the most recent forecast file. The additional analysis file from 11 days prior to the date of the solution is added to aid in smoothing the data, as described later. Joining of the analysis and forecast data into a single time series requires an estimate of the bias between the models used in the MRF/AVN system to produce the analysis and forecast data. A two-point forward extrapolation of the last two entries in the most recent analysis file is employed to estimate the AAM analysis value $6 \mathrm{~h}$ into the future. Likewise, a two-point backward extrapolation of the first two forecast values gives an estimate of the prediction for the same epoch. The difference between these two estimates is taken as the bias between the two models and applied as a correction to the forecast series.

This study also re-examined the performance of the AAM computed with the Inverted Barometer correction applied and the AAM time series without the IB correction by comparing the UTAAM generated by these time series to the Bulletin UT1 combination series on time scales of 5-20 days. These comparisons again indicated that the AAM computed with the IB correction was in better agreement with the Bulletin UT1 combination. Therefore, the UTAAM computed from the IB corrected AAM was chosen. 

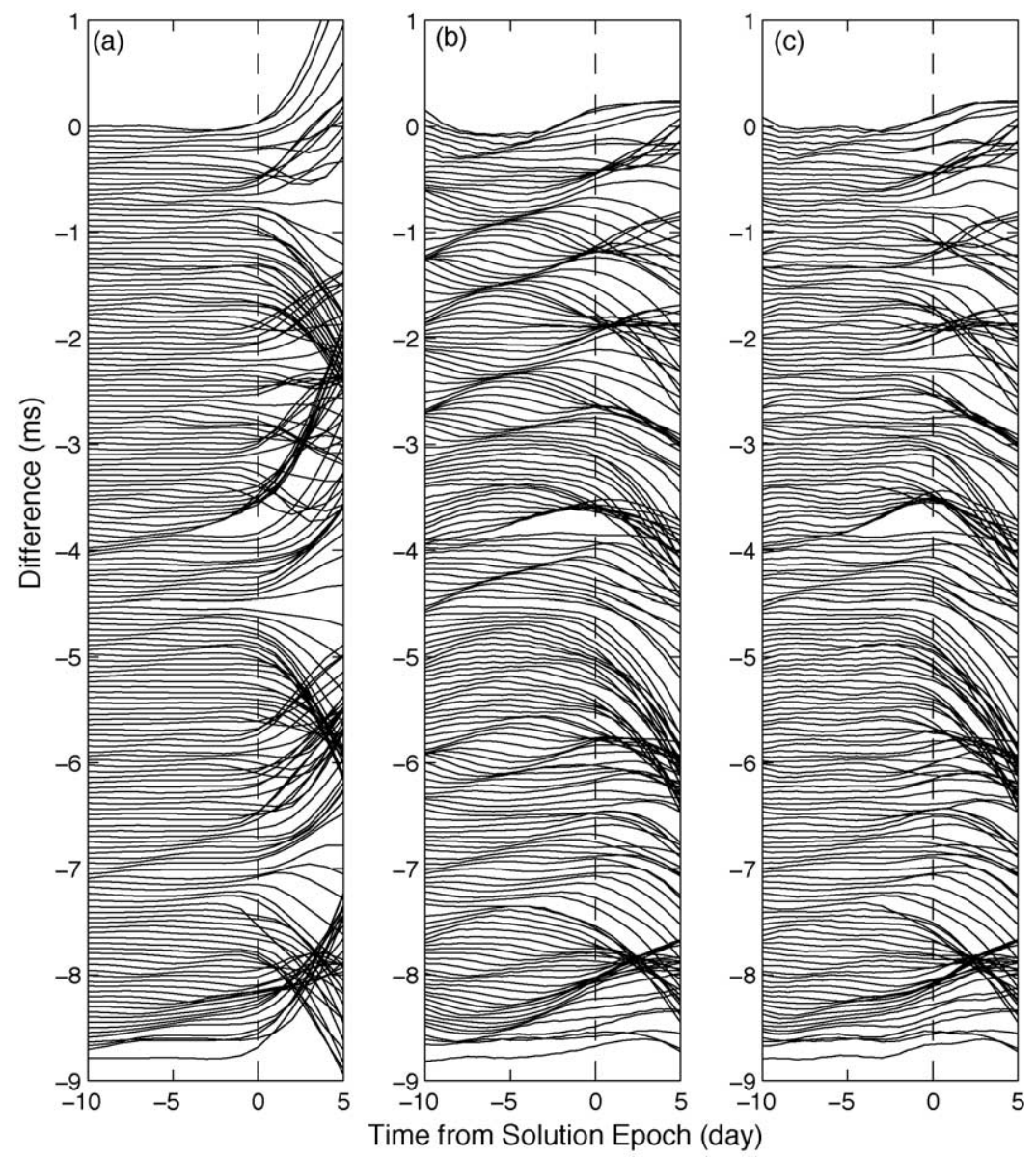

Fig. 1. The differences between the geodetic-only finals UT1R series and (a) the Daily UT1R (no-AAM), (b) the UTAAM, and (c) the UTAAM case 3 for the period from February (top) to August (bottom) 2001. For (b) and (c), a linear trend was removed from the 15-day series. This linear trend was computed using the differences during the 10-day analysis period. Each day is offset by $0.05 \mathrm{~ms}$.

In our earlier effort to use UTAAM in the IERS Bulletin UT1 combination, the analysis data file did not contain AAM data at the 24-h epoch. This required the estimation of an UTAAM value at the missing epoch as well as the bias between the forecast and analysis data. This was a large source of the error in our earlier effort that is now greatly reduced with the introduction of AAM analysis data at the 24-h epoch.

To reduce possible aliasing of higher frequency variability into the lower frequencies, the four times daily analysis data are smoothed with 5-point normalized Hanning window before the time series is subsampled every $12 \mathrm{~h}$. After examining a few different windows, a Hanning window was chosen because of its well know characteristics with respect to the leakage of higher frequency power into the lower frequencies. The bias-corrected forecast data series is joined to the end of this sub-sampled analysis series. The resulting 15-day AAM-derived LODR time series (LOD corrected for the well known zonal tidal effects, Yoder et al., 1981) is again smoothed with a 5-point normalized Hanning window. This 
smoothing reduces variability with frequencies greater than the Bulletin's 0.5 cycles per day Nyquist frequency. Then, data for the 10 most recent analysis days and five forecast days are integrated to produce a 15-day UTAAM series.

The differences between UTAAM and Final geodetic UT1R indicate the presence of linear and quasiperiodic trends. Since the quasi-periodic trends present in the analysis data evidently extend into the forecast period (Fig. 1b), a least-squares sinusoid fit to the differences during the 10 days of analysis is useful in reducing the effects on the forecasts. This 10-day fit window was decided upon after examining the residuals of fits using windows ranging from 5 to 20 days. We examined three strategies for this correction: a linear trend, a linear plus sinusoidal fit, and a combination of a linear trend and sinusoidal fit with a time-dependent weighting. For this study, fits were made to the differences between the 10 days of UTAAM analysis data and Daily UT1R using least squares.

\section{Results and discussion}

Comparisons indicate that the new UTAAM is in much better agreement with the Final UT1R than the geodetic-only Daily UT1R for predictions of 2-5 days into the future. Fig. 2 and Table 2 show the standard deviation of the differences compared to geodetic-only Final UT1R for the Bulletin A geodeticonly Daily UT1R (no AAM), UTAAM after removal of a linear trend (case 1), UTAAM after removal of a linear trend and best-fit sinusoid (case 2), and UTAAM after removal of a linear trend and a best-fit sinusoid with time-dependent weighting (case 3). The time-dependent weighting causes the sinusoid values that are subtracted to vanish by the prediction at 4 days into the future. It is important to note that cases 1,2, and 3 are AAM-only solutions and they are not combined with the geodetic data used in the Bulletin. Case 3 results in the best agreement with the Final UT1R. The success of case 3 is most

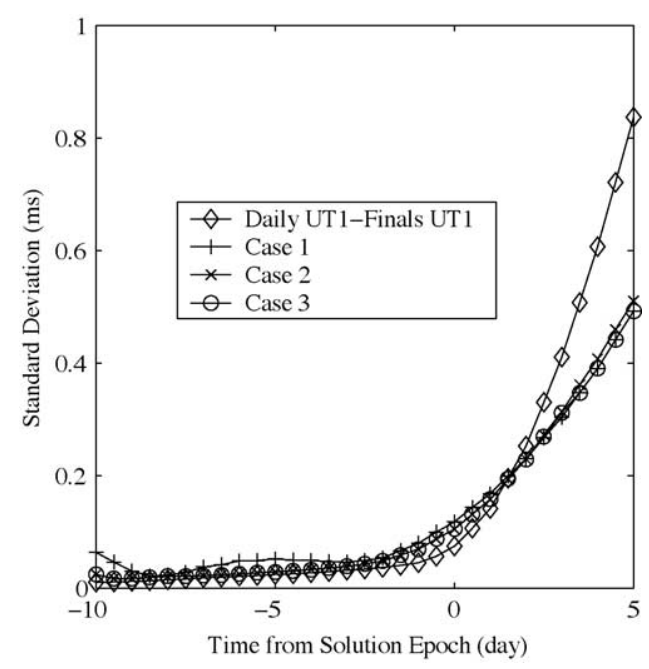

Fig. 2. The standard deviation of the differences compared to geodetic-only Final UT1R: Bulletin A geodetic-only Daily UT1R, UTAAM after removal of a linear trend (case 1), UTAAM after removal of a linear trend and best-fit sinusoid (case 2), and UTAAM after removal of a linear trend and a weighted best-fit sinusoid (case 3 ). 
Table 2

Comparison of the standard deviations for the three different cases to the EOP solution computed without the AAM time series

\begin{tabular}{lllll}
\hline Days into future & Case 1 (ms) & Case 2 (ms) & Case 3 (ms) & No AAM (ms) \\
\hline 0 & 0.118 & 0.105 & 0.105 & 0.075 \\
1 & 0.168 & 0.158 & 0.158 & 0.141 \\
2 & 0.230 & 0.229 & 0.229 & 0.253 \\
3 & 0.304 & 0.314 & 0.312 & 0.411 \\
4 & 0.390 & 0.408 & 0.390 & 0.607 \\
5 & 0.492 & 0.510 & 0.492 & 0.837 \\
\hline
\end{tabular}

Zero day into the future is the combination solution at the solution epoch and the other days are predictions into the future.

likely the result of the forecast model's drift starting to dominate the UTAAM at periods greater than $\sim 2.5$ days.

For all cases examined in this study, the predicted UTAAM, which is produced from AAM, outperformed the geodetic-only Daily UT1R for predictions greater than 2 days into the future. The case 3 strategy for handling systematic corrections was best. Fig. 1 presents the temporal performance over a 6month period comparing no-AAM (Daily UT1R), UTAAM case 1, and UTAAM case 3. A comparison of Fig. $1 \mathrm{~b}$ and c clearly shows the effectiveness of case 3 (added sinusoidal fit) in reducing the quasi-periodic variability. Table 2 and Fig. 2 indicate that the removal of a best-fit sinusoid (weighted or un-weighted) from the entire UTAAM time series is useful in reducing the AAM variability that is not present in the UT1 variability. Some drift is still present in the UTAAM for the forecast period because the fits are made using differences from the analysis period only and because of limitations in the AAM prediction model itself.

The differences between case 3 and the Final UT1R truth series have a standard deviation that is $30 \mu$ s larger than the differences between geodetic-only Daily UT1R and Final UT1R for the epoch of the combination solution, that is, at the beginning of the predictions. At the prediction time of 5-days, UTAAM case 3 has a standard derivation that is $\sim 41 \%$ smaller than the Daily UT1R solution.

The results from all the cases tested appear to indicate that the introduction of UTAAM into the combination solution, using the new analysis data files and assimilation method, will significantly improve the near-term prediction of UT1 at 5-days into the future. However, case 1 contains quasi-periodic variability that degrades the combination more for times prior to the prediction period (Fig. 2). Case 3 performs better than the other two cases at most time steps. Therefore, case 3 was chosen as the operational approach and UTAAM was reintroduced into the Bulletin A solution on 14 August 2001.

These comparisons and standard deviations are an examination of the residuals between UTAAM, an UT1-like quantity derived from AAM, and Final UT1R. When UTAAM is used in the operational Bulletin A UT1-UTC combination, only the UTAAM during the prediction period is given any significant weight. Since geodetic observations, like UTGPS, are available with minimal delay, UTAAM is not as useful before the solution epoch and may actually degrade the Bulletin A combination (see Fig. 2). Therefore, UTAAM has negligible weight in the UT1-UTC combination prior to the solution epoch but dominates the predictions. In the Freedman et al. (1994) study, the longer geodetic data latency made AAM-derived series important to both the period prior to the solution epoch and the predictions.

In addition, the operational approach substitutes an intermediate Bulletin A solution of UT1R for the previous day's Daily UT1R used in this study. The intermediate solution is closer to the solution epoch, which results in a better combination solution. The comparison of the prediction error for the 90-day 


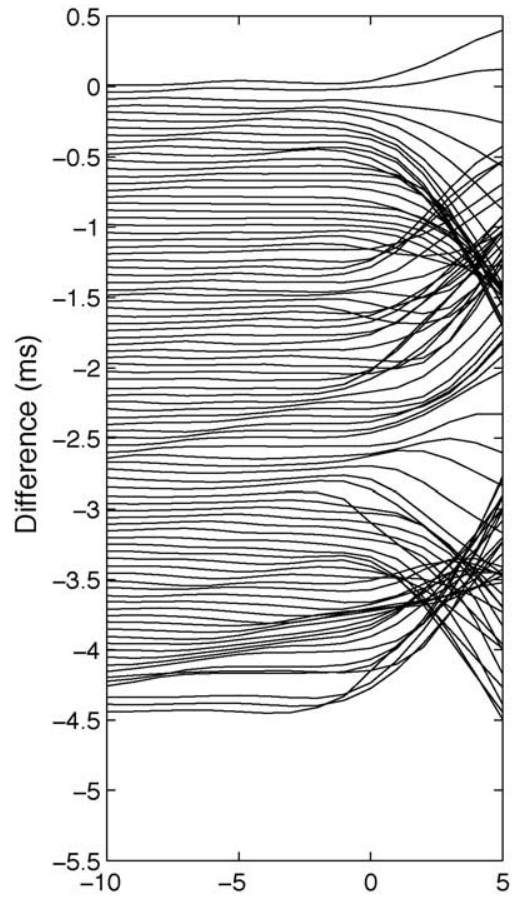

(a) Time From Solution Epoch (day)

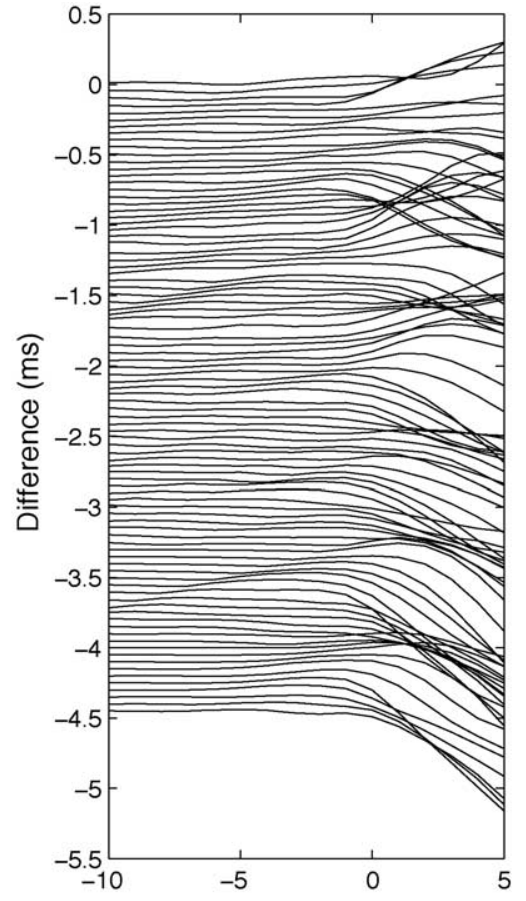

(b) Time From Solution Epoch (day)

Fig. 3. The residuals between the Finals UT1R series and: (a) the Daily UT1R (no-AAM) for the 90-day period prior to the addition of UTAAM to the UT1-UTC combination solution and (b) the Daily UT1R for the 90-day period after the addition of UTAAM to the UT1-UTC combination solution.

period before and after the reintroduction of UTAAM into the bulletin's UT1-UTC combination solution (in August 2001) shows that UTAAM reduces the prediction error by $\sim 50 \%$ at 5 days into the future (Fig. 3). A more rigorous comparison of 12-months of operational geodetic-only daily UT1R starting March 2003 and operational UT1R using both geodetic and UTAAM for the same 12-month period indicates that the introduction of UTAAM reduces prediction error by $\sim 57 \%$ at 10 days into the future. Table 3 compares the standard deviations of the differences between these two UT1R time series and the C04 UT1R time series (IERS, 1998). This comparison clearly shows that for predictions 5-30 days

Table 3

Standard deviations of the differences between the UT1-UTC time series predictions produced by the Bulletin A Daily solutions (with and without the AAM) and the C04 solution for March 2003 to February 2004

\begin{tabular}{lll}
\hline Days into future & Bulletin A without AAM (ms) & Bulletin A with AAM (ms) \\
\hline 1 & 0.091 & 0.130 \\
5 & 0.992 & 0.421 \\
10 & 2.11 & 0.840 \\
20 & 3.68 & 2.53 \\
30 & 5.22 & 4.28 \\
60 & 9.62 & 8.88 \\
\hline
\end{tabular}


Table 4

The standard deviation of the differences between UTAAM with no trends or fits removed and Final UT1R for the periods before and after the MRF/AVN model upgrade

\begin{tabular}{|c|c|c|c|c|}
\hline \multirow[t]{2}{*}{ Day of week } & \multicolumn{2}{|c|}{ Before model update } & \multicolumn{2}{|c|}{ After model update } \\
\hline & Analysis $(\mu s)$ & 5-Day forecast $(\mu s)$ & Analysis $(\mu s)$ & 5 -day forecast $(\mu s)$ \\
\hline Sunday & 469 & 1037 & 337 & 675 \\
\hline Monday & 455 & 1063 & 326 & 648 \\
\hline Tuesday & 440 & 941 & 309 & 631 \\
\hline Wednesday & 423 & 963 & 276 & 856 \\
\hline Thursday & 422 & 961 & 238 & 793 \\
\hline Friday & 442 & 1013 & 215 & 744 \\
\hline Saturday & 465 & 1023 & 211 & 702 \\
\hline
\end{tabular}

The $0 \mathrm{~h}$ epoch is used for the Analysis values and the 120 -h prediction into the future is used for the forecast values. The study period is 11 February 2001 to 11 August 2001 and the MRF/AVN system was updated on 15 May 2001.

into the future the addition of UTAAM into the combination process reduces the error in the UT1-UTC predictions. Due to the relatively short period of these two comparisons, the results should be considered qualitative and only as an estimate of relative improvement. The values presented here should not be interpreted as the actual level of Bulletin A accuracy. A longer time span of data is needed before the actual prediction accuracy levels can be estimated.

The quasi-periodic variability that is being reduced, by the removal of a best-fit sinusoid, may be the result of the limitations of current atmospheric models or oceanic effects. These modeling limitations could arise from the simplification of the equations of motion, heat transport, state, and convection that are used in the model, or they may simply be the result of modeling a continuous fluid as a discrete set of finite points.

This examination of the differences between UTAAM and UT1R has exposed changes in the performance of the atmospheric models and the effects of upgrades (see Moorthi et al., in press). Comparisons of the UTAAM before and after the upgrade on 15 May 2001 (Fig. 1b and Table 4) indicate that the new model has improved estimates of AAM variability. After the update, there was an $\sim 300 \mu$ s reduction in the standard deviation of the UTAAM residuals for the analysis data and the standard deviation of the UTAAM residuals at the 5-day prediction were $\sim 20 \mu$ s smaller. In addition, we noticed that the performance of UTAAM, prior to the upgrade, appeared to vary with respect to the day of the week. Model runs made on Sunday and Monday, which use newly assimilated observations from the previous day, typically had standard deviations that were slightly larger (Table 4). This changed after the upgrade. We have not determined the specific causes for these changes, but the upgrade did modify the model's convection, cumulus momentum mixing, transient-eddy kinetic energy loss in the upper troposphere, and the surface temperature bias. It should also be noted that the MRF/AVN model system is routinely modified to correct minor problems and these model changes are typically performed on Tuesday afternoon. These modifications may explain the trends currently observed in the AAM forecast data. The current trend in the AAM analysis data is most likely a function of the availability and/or quantity of different observation types. However, since this operational system is constantly being maintained and modified, it is difficult to determine conclusively the causes of these trends without being involved with the day-to-day operations of the MRF/AVN model system. 

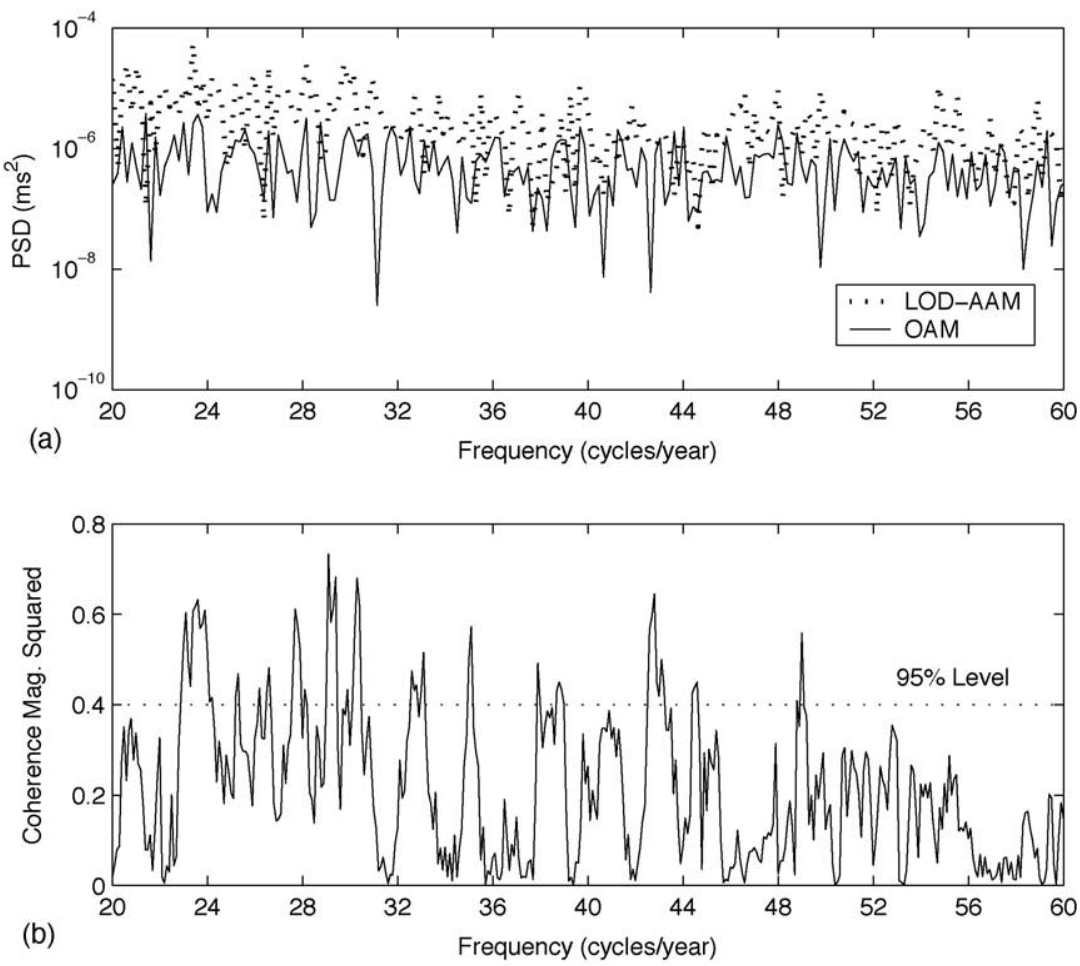

Fig. 4. (a) The power spectra of the OAM excitation function (solid line) and the variations in length of day unaccounted for by the atmosphere (dotted line). (b) The coherence magnitude squared between the OAM excitation function and the variations in length of day unaccounted for by the atmosphere time series for the years 1988-1998, using a 7-taper multi-taper spectral estimation algorithm (e.g., Chao and Eanes, 1995). The 95\% confidence level is 0.40 (dotted line).

The other possible reason for this quasi-periodic AAM variability not being observed in the actual UT1R variability is due to oceanic angular momentum (OAM) effects compensating the AAM variability. Since global ocean model data are not currently available for this time period, we compare 10 years of OAM data from 1988 to 1998, computed from the Parallel Ocean Climate Model run 4B (POCM4B) (Semtner and Chervin, 1992; Johnson et al., 1999), to variations in length of day unaccounted for by the atmosphere (LOD-AAM) using the excitation function defined by Barnes et al. (1983). NCEP reanalysis model data were used to compute the axial AAM excitation function, for the 1988-1998 period (Kalnay et al., 1996). As shown in Johnson (1998), the OAM excitation function is significantly correlated with the residual LOD-AAM at specific sub-monthly timescales. The comparison of the power spectra of the OAM excitation function and LOD-AAM indicates that both time series have power of comparable magnitudes for most of the periods between 7 and 16.6 days (Fig. 4a). Furthermore, there appears to be some significant coherence between OAM and LOD-AAM for periods in this range (Fig. 4b). However, this comparison does not allow the determination of whether the coherence is between the LOD variability not excited by the atmosphere or introduced by the atmospheric variability.

To examine the atmospheric introduced variability, we computed the multi-taper coherence between daily axial excitation functions (Barnes et al., 1983) produced from the NCEP Reanalysis (Kalnay et al., 1996) and those produced by JPL's Estimating the Circulation and Climate of the Ocean model (ECCO) 

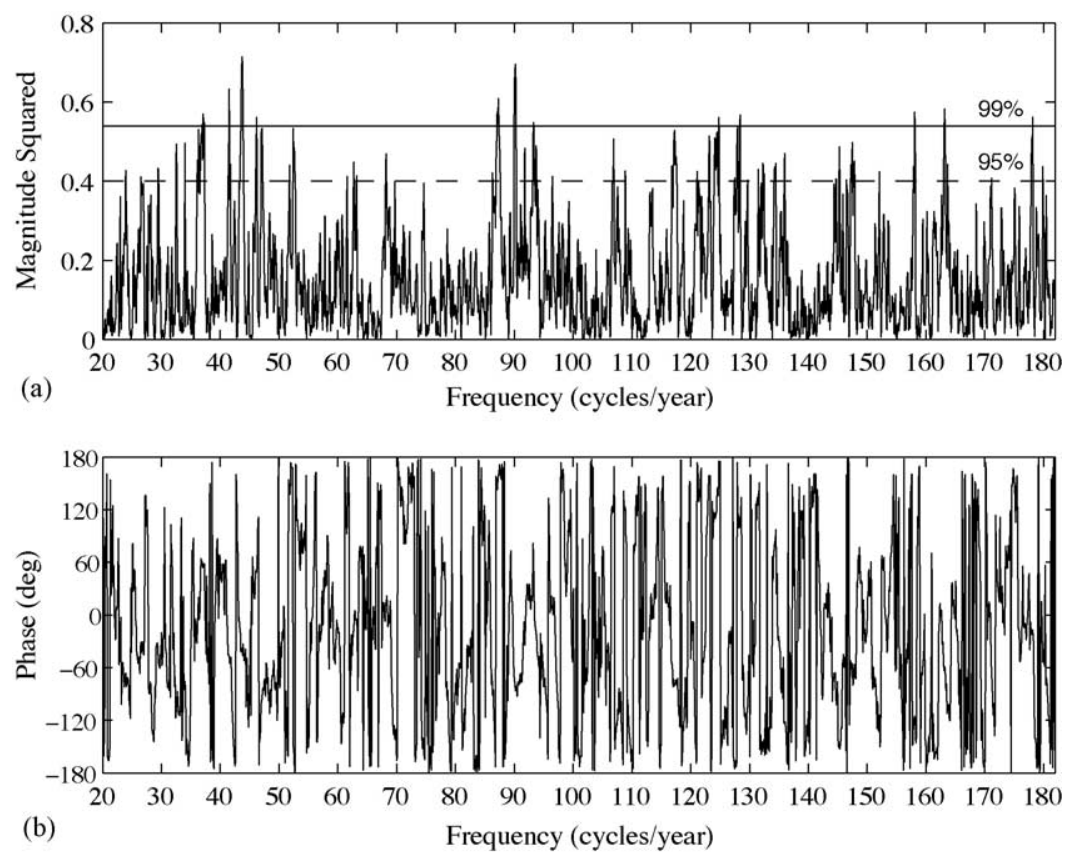

Fig. 5. (a) The coherence magnitude squared and (b) the phase between the axial components of the NCEP Reanalysis IBcorrected AAM excitation function and the ECCO model OAM excitation function for the years 1988-1998, using a 7-taper multi-taper spectral estimation algorithm (e.g., Chao and Eanes, 1995). The 95\% confidence level is 0.40 (dotted line) and the $99 \%$ confidence level is 0.54 (solid lined).

(Gross et al., 2003). Since pressure forcing was not used in driving the ECCO model, the IB-corrected AAM time series was used in this multi-taper coherence estimate. These results show significant coherence at several frequencies greater than 20 cycles/year (Fig. 5). While most of the shorter frequencies, with significant coherence greater than the $95 \%$ level, have smaller phase lags, typically less than $60^{\circ}$. Those with longer frequencies, such as $87-88$ and 124-125 cycles/year, have larger phase lags of $\sim 180^{\circ}$. Therefore, it appears that the oceans are compensating for much of this atmospheric quasi-periodic variability at periods less than 7 days. The rapid nature of this oceanic compensation appears to indicate the importance of viscosity and/or turbulent stresses on these time scales.

\section{Summary}

Comparisons with geodetic data show the presence of linear and quasi-periodic variability in the AAMderived UT1R that is not present in the actual UT1R. Left uncorrected, this atmospheric variability will limit the AAM usefulness for UT1 prediction. In the actual Earth system, the oceans probably balance most of this unaccounted atmospheric variability. However, observational data or numerical model data products do not currently exist to compensate for this variability in our operational process. Therefore, we attempt to correct for these un-modeled effects by removing a best-fit linear trend and sinusoid with a time-dependent weighting. After correcting for non-atmospheric sources, the resulting UTAAM time series significantly improves IERS Bulletin A near-term UT1-UTC predictions. Therefore, on 14 August 
2001, the UTAAM time series was incorporated back into the IERS Bulletin A UT1 combination solution using this new assimilation scheme. The reintroduction of the UTAAM information into the UT1-UTC combination solution has resulted in a significant reduction in the prediction errors at 60-days into the future. The results also indicated that the effects of recent upgrades to NCEP's operational MRF/AVN system are observable in the AAM analysis and forecast data products, and that the 15 May 2001 upgrade appears to have improved both AAM analysis estimates and predictions.

\section{Acknowledgments}

We thank G. Ranalli, editor, B. Chao and the other reviewers for their useful comments that improved this paper. Additional thanks to A. Miller and G. Long of NCEP and D. Salstein of AER, Inc., for making the NCEP AAM time series available through the IERS Global Geophysical Fluids Center's Special Bureau of the Atmosphere and JPL's J. Dickey for supporting NCEP's AAM program. This research was funded, in part, by the NASA Global Water and Energy Cycle program.

\section{References}

Barnes, R.T.H., Hide, R., White, A.A., Wilson, C.A., 1983. Atmospheric angular momentum fluctuations, length-of-day changes and polar motion. Proc. R. Soc. Lond., Ser. A 387, 31-73.

Bell, M.J., Hide, R., Sakellarides, G., 1991. Atmospheric angular momentum forecasts as novel tests of global numerical weather prediction models. Phil. Trans. R. Soc. Lond., Ser. A 334, 55-92.

Chao, B.F., Eanes, R.J., 1995. Global gravitational change due to atmospheric mass redistribution as observed in Lageos' nodal residual. Geophys. J. Int. 122, 755-764.

Freedman, A.P., Steppe, J.A., Dickey, J.O., Eubanks, T.M., Sung, L.Y., 1994. The short-term prediction of universal time and length of day using atmospheric angular momentum. J. Geophys. Res. 99 (B4), 6981-6996.

Gross, R.S., Fukumori, I., Menemenlis, D., 2003. Atmospheric and oceanic excitation of the Earth's wobbles during 1980-2000. J. Geophys. Res. 108 (B8), 2370, doi:10.1029/2002JB002143.

International Earth Rotation Service (IERS), 1998. Annual Report, Observatoire de Paris, France.

Johnson, T.J., 1998. The Role of the Ocean in the Planetary Angular Momentum Budget, Ph.D. Dissertation, University of Texas at Austin, $134 \mathrm{pp}$.

Johnson, T.J., Wilson, C.R., Chao, B.F., 1999. Oceanic angular momentum variability estimated from the Parallel Ocean Circulation Model, 1988-1998, J. Geophys. Res. 104 (B11), 25, 183-25, 195.

Kalnay, et al., 1996. The NCEP/NCAR 40-year reanalysis project. Bull. Am. Meteorol. Soc. 77, 437-471.

Kammeyer, P., 2000. A UT1-Like quantity from analysis of GPS orbit planes. Celest. Mech. Dynam. Astr. $2044,1-32$.

Luzum, B.J., Ray, J.R., Carter, M.S., Josties, F.J., 2001. Recent improvements to IERS Bulletin A combination and prediction. GPS Sol. 4 (3), 34-40.

McCarthy, D.D.(Ed.), 1996. IERS Conventions. International Earth Rotation Service Technical Note 21, Observatoire de Paris, France.

McCarthy, D.D., Luzum, B.J., 1991. Prediction of Earth orientation. Bull. Géodésique. 65, 18-21.

Morabito, D.D., Eubanks, T.M., Steppe, J.A., 1988. Kalman filtering of earth orientation changes. In: The Earth's Rotation and Reference Frames for Geodesy and Geodynamics, Proceedings of the 128th Symposium of the International Astronomical Union, Coolfont, W. Virginia, 20-24 October, 1986. Kluwer Academic, Nowell, Mass, pp. 257-267.

Moorthi, S., Pan, H.P., Caplan, P., in press. Changes to the 2001 NCEP operational mrf/avn global analysis/forecast system, NOAA Tech. Bull.

Semtner, A.J., Chervin, R.M., 1992. Ocean general circulation from a global eddy-resolving model. J. Geophys. Res. 97, 5493-5550.

Yoder, C.F., Williams, J.G., Parke, M.E., 1981. Tidal variations of Earth rotation. J. Geophys. Res. 86, 881-891. 